\title{
COMPETENCIAS METACOGNITIVAS EN LOS ESTUDIANTES UNIVERSITARIOS DE LA LICENCIATURA DE MATEMÁTICA EN LA UNIVERSIDAD POPULAR DEL CESAR
}

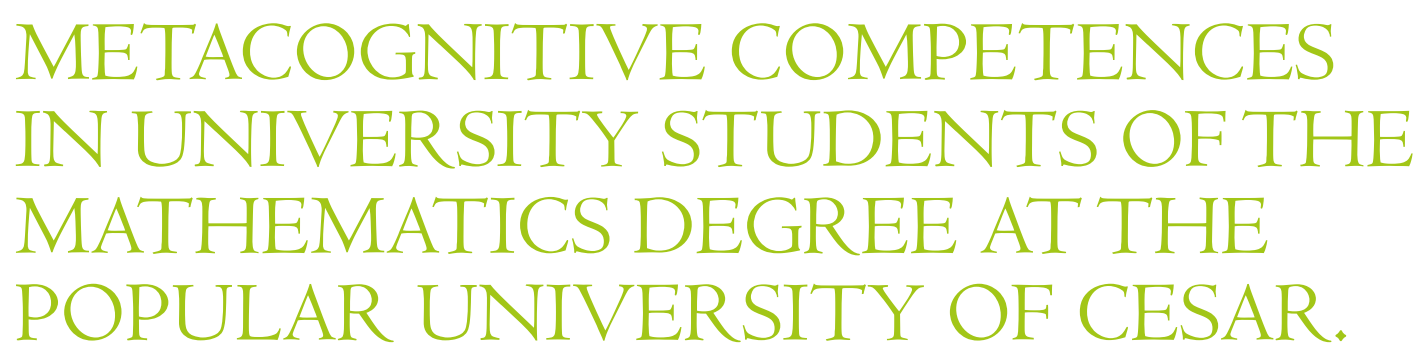

\author{
Br. Dulberto Herrera Ramos ${ }^{2}$ \\ Br. Moisés Elías Añez Oñate ${ }^{3}$
}

Dra. María de las Mercedes Colina ${ }^{4}$

Dr. Teovaldo García Romero ${ }^{5}$

Universidad Popular del Cesar, estudiantes de los semilleros de investigación pedagogía activa, directora

Dra. María Mercedes Colina

y semillero didáctica de las matemáticas, cuyo director es el

Dr. Teovaldo García Romero.

1 Trabajo desarrollado con Estudiantes de los semilleros de investigación Pedagogía activa, directora Dra. María Mercedes Colina y Semillero Didáctica de las matemáticas, cuyo director es el Dr. Teovaldo García Romero.

$2 \quad$ dherrrera@unicesar.edu.co Estudiante en formación la Licenciatura de Matemática y física joven investigador del Semillero de investigación Pedagogía al día y de didáctica de las matemáticas de la Universidad Popular del Cesar, Colombia. https://orcid.org/0000-0001-5140-5807

3 meanez@unicesar.edu.co Estudiante en formación la Licenciatura de Matemática y física joven investigador del Semillero de investigación Pedagogía al día y de didáctica de las matemáticas de la Universidad Popular del Cesar, Colombia $4 \quad$ mcolina@unicesar.edu.com Dra. en ciencias gerenciales. Investigador del grupo de investigación interdisciplinario pensamiento numérico, políticas públicas de ciencia, tecnología e innovación, medio ambiente, problemas de la educación Latinoamericana y del Caribe, categoría A, según Minciencias, directora del semillero de investigación pedagogía al día. Docente Universidad Popular del Cesar, https://orcid.org/0000-0002-6018-2884.

5 teovaldogarcia@unicesar.edu.co Investigador Universidad Popular del Cesar, docente Universidad Popular del Cesar, categoría Titular, investigador Senior, director del semillero de investigación didáctica de las matemáticas y líder grupo de investigación interdisciplinario pensamiento numérico, políticas públicas de ciencia, tecnología e innovación, medio ambiente, problemas de la educación Latinoamericana y del Caribe, categoría A, según Minciencias. Orcid ld https://orcid.org/0000-0002$\underline{6398-5113}$ 


\section{RESUMEN}

El objetivo de la investigación es analizar las competencias metacognitivas en los estudiantes universitarios de la licenciatura de matemática en la Universidad Popular del Cesar. La variable de estudio quedo definida como competencias metacognitivas, sustentada por Flavell (1996) Arias, Zegarra y Velarde (2014) Piña y Alfonso (2019), entre otros, fundamentada por el paradigma positivista, con un diseño no experimental, transaccional y de campo descriptivo; de naturaleza cuantitativa correspondiente a un proceso de codificación, tabulación y análisis estadístico; con una población de 24 estudiantes cursantes del último semestre de la licenciatura de matemática y física a los cuales se les aplico un cuestionario virtual tipo Likert con escala de alternativas de que permitieron establecer parámetros de medición de la variable. En el análisis de los resultados se evidenció la necesidad que tienen los estudiantes de implementar estrategias metacognitivas que impulsen su crecimiento integral, preparándolo para el campo profesional educativo con herramientas de planificación, control y evaluación que fortalezcan sus habilidades al servicio del logro de sus objetivos y los del contexto.

PALABRAS CLAVES: Competencias metacognitivas, estudiantes universitarios.

\section{ABSTRACT}

The objective of the research is to analyze the metacognitive competences in the university students of the mathematics degree at the Popular University of Cesar. The study variable was defined as metacognitive competences, supported by Flavell (1996) Arias, Zegarra and Velarde (2014) Piña and Alfonso (2019), among others, based on the positivist paradigm, with a non-experimental, transactional and field design descriptive; quantitative in nature corresponding to a process of coding, tabulation and statistical analysis; With a population of 24 students in the last semester of the mathematics and physics degree to which a virtual Lickerttype questionnaire was applied with a scale of alternatives that allowed to establish measurement parameters of the variable. In the analysis of the results it was evidenced the need for students to implement metacognitive strategies that promote their integral growth, preparing them for the educational professional field with planning, control and evaluation tools that strengthen their skills at the service of achieving their objectives and those of the context.

KEYWORDS: Metacognitive competences, university students.

\section{INTRODUCCIÓN}

La globalización con los cambios a nivel político, económico, cultural y tecnológicos viene demarcando la era del conocimiento, impactando el sistema educativo con transformaciones que arropan las universidades; lo cual demanda en los estudiantes afianzar su aprendizaje con aptitudes y competencias que se extiendan más allá del conocer, evolucionando en el área espiritual, ética y estética, con sentido de vida; adaptándose a las exigencias del entorno con flexibilidad, de tal manera que activen los procesos de pensamiento de orden superior, considerando, específicamente la resolución de problemas.

Cabe destacar que, autores como Piña y Alfonso (2019), afirman que los estudiantes latinoamericanos indistintamente de la carrera que cursen carecen de habilidades metacognitivas, mientras que las investigaciones de Arias, Zegarra y Velarde (2014) determinaron la existencia de niveles bajos de conocimiento y regulación del aprendizaje; por su parte Correa Castro y Lira (2014) muestran que no tienen el hábito de reflexionar ni regular sus conocimientos. 
Aunado a ello, la crisis provocada por el COVID-19, el aislamiento social, la enseñanza remota, entre otros factores han provocado traumas, cansancio y agotamiento mental tanto en los docentes como en los estudiantes universitarios, condicionando el aprendizaje a un estado de apatía, aburrimiento y en oportunidades abandono del sistema, no solo por la falta de herramientas digitales, sino también por ausencia de estrategias que orienten el desarrollo metacognitivo.

Cabe destacar que, bajo los preceptos de la teoría constructivista, cognitiva y humanista, el estudiante puede edificar el propio conocimiento partiendo de su propia forma de ser, pensar e interpretar la información, conservando una actitud centrada en el beneficio de lo humano, en su pleno desarrollo y bienestar, incluso desde una mirada biológica, psicológica, social, cultural y espiritual.

Aportando a estas interpretaciones, Jiménez (2020), refiere que los avances de la neurociencia propician el desarrollo de las capacidades mentales, dinamizando la metacognición de los estudiantes universitarios; considerando que el aprendizaje se genera a través de la actividad del cerebro y del sistema nervioso central donde aparece la sensación, integración y respuesta en equilibrio como unidad básica y funcional de todo ser vivo.

Shimamura (2008), asevera que la metacognición como proceso depende de diferentes actividades, acciones y funciones cognoscitivas llevadas a cabo por los estudiantes universitarios para lograr alcanzar sus metas; es decir, el estudiante tiene la capacidad de utilizar herramientas intelectuales que ya están interiorizadas por él, facilitándole la obtención de información, para procesarla, evaluarla y transformarla validando lo que sabe y lo que tiene, haciendo su propio análisis y autorregulación de su aprendizaje intelectual; de allí que, la metacognición definida por Mateos
(2001) es una habilidad que integra los procesos $\mathrm{u}$ operaciones cognitivas del ser humano para adaptarse al medio y resolver situaciones.

Para Flavell citado por Mateo (2001, p22) "el desarrollo cognitivo no solo implica un aumento de la complejidad estructural del sistema cognitivo, sino también del acceso consciente". Por tanto, un estudiante universitario requiere concientizar sus capacidades y habilidades para lograr la regulación activa de sus competencias con resultados favorables en su desempeño académico, así como en su actuar que lo conduzcan a tomar decisiones en pro de solucionar los problemas acontecidos en el entorno

Desde las interpretaciones anteriores, la importancia de desarrollar competencias metacognitivas en los estudiantes universitarios se relaciona directamente con la eficiencia académica, el aprendizaje autorregulado, el desarrollo del pensamiento crítico, entre otros.

Cabe destacar que, las trasformaciones por las que está atravesando el mundo y Latinoamérica, como sistema se reflejan en Colombia y por ende en sus casas de estudios universitarias, en donde el foco de la enseñanza recae en el conocimiento específico de carácter técnico pasando a un segundo plano el desarrollo de competencias metacognitivas, como habilidad necesaria para afianzar la inteligencia relacional y mejorar los entornos del aprendizaje.

Al respecto, Núñez (2015) indica que el sistema universitario del país está en la obligación de voltear la mirada hacia los currículos de carreras profesionales ofertadas en el presente, en función de desarrollar y potenciar las destrezas de los estudiantes con base a un proceso de aprendizaje metacognitivo que le permitirá solucionar problemas, adaptarse a entornos cambiantes, responder a desafíos y tomar decisiones oportunas que generen la 
construcción de un conocimiento socio afectivo que edifique su desarrollo profesional.

Debe señalarse, que el Decreto Nacional 2566 del 2003, modificado por el Decreto Nacional 2170 de 2005, estableció los requisitos y las condiciones mínimas de calidad que faciliten el desarrollo de programas académicos de educación superior, resaltando el alcance de la autonomía intelectual; así como, el valor de las personas con capacidad para tomar decisiones en el curso de su existencia hasta que culminen sus proyectos de formación profesional.

Sin embargo, a pesar de la existencia de lineamientos que buscan impulsar el crecimiento integral del estudiante, dentro de la Universidad popular del Cesar, específicamente de la licenciatura de matemática y física, se evidencia a través de observaciones no estructuradas que los mismos presentan problemas de planificación, control, evaluación y mejora de sus procesos, limitándose solamente a conceptualizar el conocimiento con ausencia de herramientas que le permitan solventar situaciones que ameriten toma de decisiones involucrando el pensamiento crítico y creativo.

Esta realidad, mantiene desalineada a la Universidad Popular del Cesar de los objetivos que se plantea la Unesco en la meta 4,7 sobre el desarrollo sostenible hasta el año 2030 , donde se busca que el estudiante tenga en cuenta las competencias que el mercado laboral requiere de cara a escenarios innovadores provocados por las transformaciones ocurridas en los últimos años, haciéndose necesario que los centros educativos introduzcan nuevos esquemas de enseñanza orientados hacia la adquisición de conocimientos tanto teóricos como prácticos que integren el desarrollo cognitivo, socioemocional y conductual del individuo.

En función de ello, el aprendizaje integral de los estudiantes de la licenciatura de matemática y física no solo depende de los esquemas educativos de la Universidad, sino que también requiere que estos futuros profesionales se hagan responsables del desarrollo de sus competencias metacognitivas, reconociendo sus capacidades y experiencias para asimilar un proceso de formación que coadyuva a resolver sus propios problemas y los del entorno (De Zubiría, 2007).

De allí que Botero et al (2017), afirman que una de las formas más efectivas que posee el estudiante para adquirir herramientas metacognitivas, es poniendo en práctica estrategias de aprendizaje autónomas que le permitan responder ágilmente a sus necesidades, las de los contextos universitarios y las requeridas en su ejercicio profesional.

Visto desde estas perspectivas, el joven universitario debe tomar consciencia sobre la adquisición de nuevas formas de aprendizaje que lo conduzcan a capacitarse para resolver las situaciones cotidianas, comunicarse y convivir en una sociedad demandante y competitiva, por ello las competencias metacognitivas se convierten en un requisito indispensable para alcanzar los saberes específicos, comprendiendo tanto el desarrollo social como el emocional que implica estructurar su arquitectura mental para generar procesos de autogestión y el control ejecutivo que fortalecerá su comprensión, evaluación, generación de información, toma de decisiones, así como la resolución de problemas que infiere en la conducta sobre lo que se dice y lo que se hace.

Por ello, resulta positivo transformar las bases del aprendizaje de los estudiantes de la licenciatura de matemática y física, en función de ponderar los procesos de planificación, control, evaluación y mejora para impulsar de manera estratégica su compromiso por reforzar su conciencia cognitiva, con la capacidad de aprender a pensar en forma dinámica y funcional facilitándole la apropiación 
de recursos, habilidades y herramientas para afrontar los retos de la realidad profesional a la cual se verán enfrentados, por cuanto hoy por hoy en la enseñanza de la matemática de acuerdo a Sandoval (2014), el aprendizaje será estratégico en tanto el estudiante tenga recursos metacognitivos para administrar todo lo referido al hecho de aprender.

De acuerdo a lo expuesto anteriormente, surge la pregunta ¿de qué manera se desarrollan las ccompetencias metacognitivas en los estudiantes universitarios de la licenciatura de matemática y física en la Universidad Popular del Cesar?

Sobre el asunto, para generar una transformación significativa que apueste por el crecimiento de los procesos de aprendizaje metacognitivos, se plantea como objetivo general analizar las competencias metacognitivas en los estudiantes universitarios de la licenciatura de matemática y física en la Universidad Popular del Cesar y como objetivos específicos los siguientes:

a) Identificar los tipos de competencias metacognitivas para el aprendizaje de los estudiantes universitarios de la licenciatura de matemática y física en la Universidad Popular del Cesar.

b) Describir los componentes metacognitivos para el aprendizaje de los estudiantes universitarios de la licenciatura de matemática y física en la Universidad Popular del Cesar.

c) Proponer estrategias metacognitivas para el aprendizaje de los estudiantes universitarios de la licenciatura de matemática y física en la Universidad Popular del Cesar.

\section{CUERPO Y DESARROLLO}

\section{Competencias Metacognitivas.}

La UNESCO (2005) define las competencias como el conjunto de comportamientos socio afectivo y habilidades cognoscitivas, psicológicas, sensoriales y motoras que permiten llevar un adecuado desempeño, una función, una actividad o una |tarea. Por su parte Tejada y Navío (2005), afirman que las competencias están enmarcadas en las acciones coordinadas de un individuo donde están presentes los conocimientos, procedimientos y actitudes en el desempeño de una tarea. En este orden Ledezma (2010), hace alusión a las competencias metacognitivas, afirmando que, si bien es cierto el individuo tiene conocimientos sobre sus propios procesos cognitivos, también está dotado de capacidades que puede direccionarse para solucionar los problemas existentes en la cotidianidad, lo que origina que sus acciones tengan un aprendizaje significativo.

Bajo este escenario, los estudiantes de la licenciatura de matemática, inmersos en las exigencias de su formación como futuros docentes, deben ser conscientes de sus competencias metacognitivas, pues les permiten desarrollar los procesos de conocimientos internos y externos, así como construir estrategias que le faciliten apropiarse de sus habilidades y hacerles frente a las dificultades del entorno.

Incluso, pueden diseñar su futuro, planificando y evaluándolo en función de regular sus acciones antes durante y después de una actividad, lo que le da holgura para diseñar y utilizar estrategias acordes con la realidad, haciendo una transferencia gradual sobre su aprendizaje a otros espacios, reforzando su autonomía y autorregulación. (Méndez, 2013) y (Mateos, 2001).

\section{Tipos de competencia metacognitivas.}

Los tipos de competencias demarcan un conjunto de capacidades, procedimientos y actitudes que acompañan al individuo para 
desarrollarse en un área específica académica o profesional, apoyadas en valores sociales y culturales a través de la comunicación y cooperación permanente que le permiten autorregular su propia conducta en función de tomar de decisiones con responsabilidad y aumentar los niveles de motivación cuando se logra el objetivo propuesto

\section{Competencia de Planeación. Esta competencia} está orientada a regular las acciones del aprendizaje, facilitando al estudiante de la licenciatura en matemática identificar, seleccionar, organizar y procesar la información que requiere de manera pertinente, verificando el que, cómo y cuándo debe hacer la tarea asignada, lo que implica activar su memoria recordando sus conocimientos previos aplicando estrategias para validar sus resultados.

Para De Miguel (2006) y Méndez (2013), la competencia de planeación, recae en la visión del estudiante universitario para armar las estrategias desde el comienzo de su aprendizaje hasta el final, por ello requiere estar en conocimiento de lo que quiere fijando las metas y objetivos, asignando el tiempo, así como también un plan de ejecución para alcanzar su propósito de aprendizaje.

Competencia de autorregulación. Referida a la capacidad para analizar cuáles son las características de una actividad, lo cual permite optimizar los procesos en función del producto que se quiere lograr, Ormrond (2008). Para alcanzar esta competencia se requiere establecer objetivos, planificar en función de los recursos, automotivación, controlar la atención evitando los distractores, apropiarse del material, auto controlarse, autoevaluarse y auto reflexionar.

En este orden, cuando el estudiante de matemática está consciente de su rol como futuro profesional de la docencia, anticipa su estrategia de aprendizaje, elabora su planificación, considerando la forma del como la va a ejecutar, monitorea sus avances asumiendo el error como parte del proceso que lo invita a tomar correcciones y avanzar; por último, reflexiona sobre sus resultados siendo flexible para mejorar de manera continua.

Competencia de autocontrol. De Miguel (2006), afirma que esta competencia tiene su centro en la motivación y el auto refuerzo. Al considerar los actuales escenarios donde está presente la virtualidad se requiere que el estudiante de matemática comprenda las tareas a realizar para lo cual es indispensable que efectué preguntas que generen claridad en los procedimientos que se deben seguir, de esta manera alcanzar los resultados deseados, aumentando la confianza en sus habilidades, disminuyendo la ansiedad y fortaleciendo su autoestima y así auto gestionar las dificultades que se presenten.

\section{Competencias de autoevaluación del} aprendizaje. Desde el punto de vista didáctico, esta competencia es pilar fundamental antes, durante y después del proceso de aprendizaje, pues permite que el estudiante de matemática como futuro profesional de la docencia pueda medir y valorar la práctica en sí mismo, para luego transferir sus conocimientos a sus pupilos. Bajo este escenario García, et al (2006), afirma que las ventajas que tiene el estudiante universitario cuando se hace consciente de esta competencia radica en la posibilidad de evaluar continuamente sus logros, reducir el tiempo de ejecución en la entrega de las tareas, bajar el nivel de ansiedad por lograr los resultados, flexibilizar sus exigencias individuales considerando su ritmo y retroalimentarse para innovar y adaptarse eficazmente a los cambios que se presenten hoy, así como en su futuro como profesional de la docencia.

\section{Competencias tecnológicas. Es la capacidad} que tienen los estudiantes universitarios para acceder al conocimiento que se encuentra en 
línea, en este particular hay que aclarar que las estrategias presenciales distan en su forma de aquellas que se utilizan en la virtualidad. De allí que, la búsqueda correcta de la información determina el nivel de conocimiento.

Area, Gros y Marzal (2008) afirman que para desarrollar estas competencias se requiere: Alfabetización en lectoescritura y cultura impresa, en lenguaje y cultura audiovisual, en tecnologías y cultura digital y la alfabetización informacional, para concretar una información actualizada ajustada a las necesidades $y$ exigencias del medio. Cabe destacar, que cuando el estudiante de la licenciatura de matemática está en presencia de la automotivación tiene la disposición de adaptarse a la realidad de los entornos virtuales de manera efectiva y consciente de la modificación de sus estrategias para tener un aprendizaje significativo.

Atendiendo a lo anterior, las competencias descritas, se pueden evidenciar con indicadores de gestión que impacten la autogestión del aprendizaje de los futuros licenciados de matemática; puesto que, una vez que se hace consciente de cuál es el proceso que se requiere para apropiarse de sus habilidades, estarán en la capacidad de aplicar conocimientos y procedimientos en su hacer pedagógico; así como, considerar los elementos culturales que acompañan su desempeño, los cuales tienen influencia tanto en la creatividad como en la disposición para estar en una autoevaluación de mejora continua, modificando su actitud y conducta atendiendo las propias exigencias y a las del entorno educativo donde se desenvuelven.

\section{Componentes metacognitivos.}

Son elementos indispensables para la construir, dirigir y controlar el aprendizaje, otorgándole un significado al proceso que se realiza en la edificación del mismo como producto de un pensamiento donde adquiere sentido planificación, supervisión y evaluación del acto mental. Mateos (2001), basado en Flavell (1996) los describe como:

\section{Conocimiento declarativo (referido al qué)}

Representado en primer lugar por la consciencia que tiene el individuo sobre qué factores interactúan en él y afectando los procesos y los resultados de las actividades cognitivas. Entre los cuales se encuentran los factores personales, referidos a los conocimiento sobre las creencias de sí mismo como persona, así como también las capacidades y limitaciones que se han almacenado en la memoria potenciando o limitando la realización de las tareas; de igual manera, este componente considera 3 aspectos importantes: la experiencia que demarca el progreso a la meta con los pensamientos, sensaciones y sentimientos que acompañan la actividad cognitiva; las propias habilidades orientadas hacia las condiciones mínimas que debe tener el estudiantes para desarrollar habilidades que aporten solución a los problemas, donde se incluye la experticia para seleccionar las actividades adecuadas y por último el conocimiento acerca de las capacidades de la persona para comprender y reconocer procedimientos alternativos que aborden una tarea desde otro punto de vista, reflexionando conscientemente sobre los obstáculos que se presentan y las distintas soluciones que se aplican para alcanzar los objetivos.

En un segundo término el conocimiento declarativo está referido a la tarea, donde influye en el conocimiento que tienen los estudiantes para la ejecución de la misma, estando representado, en un primer lugar por el nivel de dificultad de la acción que se ejecuta, donde se requiere un equilibrio entre la capacidad propia, la coherencia, las estrategias y el como el estudiante evaluara los objetivos que se propone; en un segundo lugar por la atención en la ejecución de la tarea, donde interviene la 
motivación y la concentración; y por último, el cómo se aborda la tarea, lo cual quiere decir que debe tener herramientas adecuadas para realizar las actividades planificadas. (Mateos, 2001)

En este sentido, el conocimiento declarativo para finalizar esta referido a la estrategia que hace referencia "al conocimiento de la efectividad relativa de los procedimientos relativos para abordar una tara" (Mateos, 2001 p. 23), es decir, es la capacidad que posee el estudiante de buscar diferentes formas efectivas de plantearse el abordaje de una tarea visualizando el panorama completo desde el inicio del desarrollo de la actividad, evaluando previamente los distintos escenarios que se puedan presentar, haciendo consciencia del cuándo, dónde y cómo aplicar las técnicas para focalizar los puntos neurálgicos del objetivo.

Este conocimiento estratégico puede ser de naturaleza cognitiva, de comprensión dirigida o de comprensión para progresar en la actividad y de regulación para verificar el cumplimiento del objetivo.

De acuerdo a lo anterior, reconocer el componente declarativo de la metacognición les permite a los estudiantes de la licenciatura de matemática tener consciencia sobre la tarea que van a realizar, de forma tal, que tengan la oportunidad de planificarse en función del logro de las metas, con el valor agregado que da el conocimiento sobre las acciones tendentes a solucionar los obstáculos que se presenten.

\section{Conocimiento procedimental (referido al cómo)}

Este componente, de acuerdo a Mateos (2001), describe el proceso de una tarea, la regulación de la misma y la verificación para poder replantear asignación de recursos y estrategias; es decir, representa los lineamientos, procedimientos y tareas que regulan la forma de ejecutar las asignaciones de los estudiantes, estando caracterizada por comprender el cuándo y el porqué de la acción.

En este sentido, los componentes de la metacognición le permiten al estudiante de la licenciatura de matemática y física mejorar el aprendizaje, pues por su carácter e intensión le permiten elaborar, desarrollar y evaluar un plan de acción dinámico alineado a sus intereses, que, haciendo énfasis en lo pedagógico, fortalecen su formación como futuros docentes.

\section{METODOLOGÍA}

La presente investigación presenta una postura epistemológica enmarcada en el paradigma positivista de tipo analítica que de acuerdo a Hurtado (2000) tiene como objetivo analizar un evento y comprenderlo en términos de sus aspectos menos evidentes, lo cual significa desintegrar o descomponer una totalidad en todas sus partes; de igual forma, se realiza la reinterpretación de los resultados en función de los objetivos del análisis.

Para este estudio, la investigación desglosa cada elemento para llegar al análisis de las competencias metacognitivas en los estudiantes universitarios de la licenciatura de matemática en la Universidad Popular del Cesar. Cabe destacar, su carácter descriptivo puesto que se orientó a recolectar información relacionada con el estado real de las personas y fenómenos, tal y como se presenta en el contexto de la Universidad Popular del Cesar, según (Chávez, 2007)

Por su parte, Hernández, Fernández y Baptista (2010), evidencian que una investigación descriptiva está centrada en la medición de datos con la mayor precisión posible. En cuanto al diseño se ubicó en un estudio de campo no experimental, transaccional, Áreas (2006), puesto que no se manipulan las variables deliberadamente y se 
observan los sujetos o fenómenos de estudio en un ambiente natural y en un tiempo determinado.

Para el análisis de los datos se trabaja con una población finita constituida por 24 estudiantes, Parra (2003), con edades comprendidas entre los 18 y 23 años, 10 de sexo femenino y 14 de sexo masculino, todos activos, con asignaturas aprobadas y cursantes del último semestre de la licenciatura de Matemática y Física de la Universidad Popular del Cesar.
Para el abordaje de la recolección de la información, de acuerdo a Hernández, Fernández y Baptista (2010), se empleó una encuesta virtual en una escala de medición de actitudes tipo Likert, con 21 afirmaciones en las que principalmente interesa la opinión que expresa la persona entrevistada, con un baremo descrito por opciones de respuestas (5) Siempre, (4) Casi siempre, (3) Algunas veces (2) Casi nunca, (1) Nunca, el cual se le aplico a la muestra

\section{Cuadro número 1}

Baremo para la interpretación de la media.

\begin{tabular}{|c|c|c|c|}
\hline Alternativas & Categoría & Límites & Resultado \\
\hline Siempre & Muy alto dominio & $4.21 \geq \mathrm{X}<5.00$ & \multirow{2}{*}{ Favorable } \\
\hline Casi siempre & Alto dominio & $3.41 \geq \mathrm{X}<4.20$ & \\
\hline Algunas veces & Moderado dominio & $2.61 \geq \mathrm{X}<3.40$ & Neutral \\
\hline Casi nunca & Bajo dominio & $1.81 \geq \mathrm{X}<2.60$ & \multirow{2}{*}{ No favorable } \\
\hline Nunca & Muy bajo dominio & $1.00 \geq \mathrm{X}<1.80$ & \\
\hline
\end{tabular}

Fuente: Elaboración propia

Como se puede inferir, el procedimiento empleado para tabular y codificar los datos permite el análisis de los mismos, así como su organización, determinando el número de los casos que corresponden a las categorías de las respuestas. Por tanto, la estadística utilizada en la presente investigación permite evaluar los resultados, desde sus dimensiones y sus respectivos indicadores. (Hernández, Fernández y Baptista, 2010)

\section{RESULTADOS DE LA INVESTIGACIÓN}

Posterior a la recolección de la información mediante la aplicación del cuestionario virtual a 24 estudiantes perteneciente al último semestre de la licenciatura de matemática y física, se elaboró una matriz para analizar los datos obtenidos.

Variable: Competencias Metacognitivas

Dimensión: Tipos de competencias Metacognitivas 


\begin{tabular}{|c|c|c|c|c|c|c|c|c|}
\hline & & Prom & \multicolumn{6}{|c|}{ Frecuencias } \\
\hline \multicolumn{2}{|c|}{ Promedio general } & 3,13 & Abrev. & $s$ & cs & AV & CN & $\mathrm{N}$ \\
\hline \multirow{5}{*}{$\begin{array}{l}\text { Tipos de competencia } \\
\text { metacognitivas }\end{array}$} & $\begin{array}{l}\text { Competencia de } \\
\text { Planeación }\end{array}$ & 3,94 & $\begin{array}{l}\text { FA } \\
\text { FR }\end{array}$ & $\begin{array}{r}19 \\
26 \%\end{array}$ & $\begin{array}{c}36 \\
\mathbf{5 0 \%}\end{array}$ & $\begin{array}{c}11 \\
15 \%\end{array}$ & $\begin{array}{c}6 \\
8 \%\end{array}$ & $\begin{array}{l}0 \\
0 \%\end{array}$ \\
\hline & $\begin{array}{l}\text { Competencia de } \\
\text { autorregulación }\end{array}$ & 3,72 & $\begin{array}{l}\text { FA } \\
\text { FR }\end{array}$ & $\begin{array}{c}22 \\
31 \%\end{array}$ & $\begin{array}{c}20 \\
28 \%\end{array}$ & $\begin{array}{c}21 \\
29 \%\end{array}$ & $\begin{array}{c}6 \\
8 \%\end{array}$ & $\begin{array}{c}3 \\
4 \%\end{array}$ \\
\hline & $\begin{array}{c}\text { Competencia de } \\
\text { autocontrol }\end{array}$ & 2,96 & $\begin{array}{l}\text { FA } \\
\text { FR }\end{array}$ & $\begin{array}{c}17 \\
24 \%\end{array}$ & $\begin{array}{c}9 \\
13 \%\end{array}$ & $\begin{array}{c}11 \\
15 \%\end{array}$ & $\begin{array}{c}24 \\
33 \%\end{array}$ & $\begin{array}{c}11 \\
15 \%\end{array}$ \\
\hline & $\begin{array}{c}\text { Competencias de } \\
\text { autoevaluación } \\
\text { del aprendizaje }\end{array}$ & 2,47 & $\begin{array}{l}\text { FA } \\
\text { FR }\end{array}$ & $\begin{array}{c}2 \\
3 \%\end{array}$ & $\begin{array}{c}9 \\
13 \%\end{array}$ & $\begin{array}{c}21 \\
29 \%\end{array}$ & $\begin{array}{r}29 \\
40 \%\end{array}$ & $\begin{array}{c}11 \\
15 \%\end{array}$ \\
\hline & $\begin{array}{l}\text { Competencias } \\
\text { tecnológicas }\end{array}$ & 2,57 & FA & $\begin{array}{c}12 \\
17 \%\end{array}$ & $\begin{array}{l}2 \\
3 \%\end{array}$ & $\begin{array}{c}18 \\
25 \%\end{array}$ & $\begin{array}{r}23 \\
32 \%\end{array}$ & $\begin{array}{c}17 \\
24 \%\end{array}$ \\
\hline
\end{tabular}

Indicador: Competencias de planeación

El indicador competencias de planeación, arrojó un promedio de 3,94 ubicándolo con resultado favorable alto dominio, en donde el $26 \%$ y el $50 \%$ de los estudiantes encuestados indicaron que siempre y casi siempre, reconocen la importancia de emplear una estrategia de planeación cónsona con los objetivos para poder identificar, seleccionar, organizar y procesar la información que van a emplear en la realización de las tareas. Contrario a ellos, un porcentaje más bajo representado por el $15 \%$ y el $8 \%$ indican que solo algunas veces y casi nunca, respectivamente, se apoyan en herramientas de planificación para definir sus metas y objetivos, asignar recursos, tiempo e implicaciones de sus acciones, por considerarlo poco útil y que les requiere demasiado tiempo.

Indicador: Competencia de autorregulación.

Al analizar esta competencia, los datos evidencian un promedio de 3,72 ubicándolo en la categoría alto dominio resultado favorable, en donde la mayoría representado por el $31 \%$ y el $28 \%$ de la muestra señalan que siempre y casi siempre, trabajan coordinados estableciendo sus objetivos en función de los recursos que poseen, conscientes de sus limitaciones en una constante motivación, viendo el error como parte del proceso de aprendizaje y reflexionando sobre la manera de mejorar sus actuaciones. Sin embargo, el $29 \%$ indicó que tan solo algunas veces, elabora su planificación considerando sus propios recursos para lograr las metas, así como el $8 \%$ y el $4 \%$ señalaron que casi nunca y nunca, tienen en cuenta estos factores como elementos que puedan influir en su desarrollo universitario.

Indicador: Competencias de autocontrol

La competencia de autocontrol arrojo un resultado de 2,96 ubicándole en una categoría de moderado dominio con resultado neutral, donde el $33 \%$ y el $15 \%$ de los informantes de la licenciatura de matemática y física casi nunca y nunca, realizan actividades que les permitan desenvolverse en escenarios virtuales ganando más confianza en sus competencias, al contrario manifiestan un alto nivel de estrés, ansiedad y en ocasiones perdida del interés en las asignaciones; sin embargo, el $24 \%$ y $13 \%$ de los estudiantes encuestados expresaron que siempre y casi siempre, buscan auto gestionar las dificultades y retos que los entornos virtuales representan para ellos, impulsándose al logro de sus objetivos mediante el uso de preguntas que aclaren los procedimientos que deben seguir.

Indicador: Competencias de autoevaluación de aprendizaje

Esta categoría arrojo el valor más bajo, con un promedio de 2,47 categoría baja con un resultado no favorable, donde los estudiantes de la licenciatura de matemática y física que hicieron parte de la muestra en estudio en un $29 \%$ tan 
solo algunas veces, en un $40 \%$ casi nunca y en un $15 \%$ nunca logran desarrollar competencias que les faciliten realizar la medición y valoración de la práctica en sí mismo, para luego transferir sus conocimientos a sus pupilos; esta realidad se convierte en un obstáculo que impide la evaluación de su actuación en forma continua, para poder reflexionar sobre lo que están haciendo bien y lo que pueden mejorar, lo que reduciría sus niveles de ansiedad, flexibilizando la auto exigencia que en ocasiones se auto colocan como estudiantes.

Indicador: Competencias tecnológicas.

Este último indicador, competencias tecnológicas con una categoría baja presenta un promedio de 2,57 con un resultado no favorable, donde del total de los estudiantes encuestados el $32 \%$ manifiesta que casi nunca pueden acceder al conocimiento que se encuentra en línea, el $25 \%$ tan solo algunas veces ha recibido alguna formación en cultura audiovisual, tecnologías y cultura digital y el $24 \%$ a pesar que pudiesen ser conscientes de cuál es el proceso que se requiere para apropiarse de sus habilidades en un escenario virtual, presentan una desvinculación tecnológica que actualmente les obstaculiza aplicar conocimientos y procedimientos en su hacer pedagógico.

\section{Dimensión: Componentes metacognitivos}

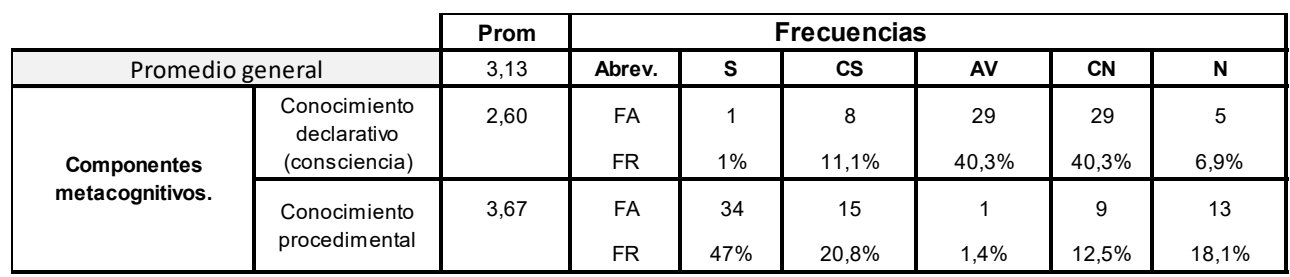

Indicador: Conocimiento declarativo (conciencia referida al qué)

Este indicador evidencia un promedio de 2,60 ubicándolo en la categoría bajo dominio con resultado no favorable, donde el $40,3 \%$ de los informantes de la licenciatura de matemática y física afirman que algunas veces tener las creencias que tienen sobre sí mismos frena su actuación profesional, llenándolos de miedo, provocando ansiedad y perdida de la propia motivación; así mismo el $40,3 \%$ de la muestra en estudio casi nunca coloca sus propias habilidades al servicio de las condiciones mínimas que deben tener para desarrollarse aportando solución a los problemas.

Indicador: Conocimiento procedimental (referido al cómo)

El indicador conocimiento procedimental, arrojo un promedio de 3,67 con un resultado favorable de alto dominio, donde el $47 \%$ y el $20,8 \%$ de los informantes siempre y casi siempre siguen lineamientos, procedimientos y la descripción de tareas que regulan la forma de ejecutar sus asignaciones, comprendiendo efectivamente la razón de ser de los mismos; sin embargo, el $12,5 \%$ y el $18,1 \%$ afirman que casi nunca y nunca, logran entender la motivación y objetivos sobre los cuales se plantean los procedimientos que deben ejecutar.

\section{ESTRATEGIAS METACODNITIVAS.}

Las estrategias metacognitivas son operaciones mentales internas que debe realizar el estudiante para mejorar el aprendizaje, las cuales tienen un carácter intencional e implican por lo tanto un plan de acción. De allí que, para autores como Flavell (1996), representan la vertiente procesual y procedimental complementarias de la vertiente declarativa del aprendizaje, como la cadena de narcoactividades $u$ operaciones mentales implicadas en el acto de aprender (comprensión, adquisición, transferencia.) (discriminar entre la información relevante e irrelevante) el modelo 
que desarrolla las técnicas de la idea principal, el resumen o abstracción.

En ellas se distinguen claramente los procesos de planificación, control y evaluación (ver figura 1). Estos orientan a la persona cuando actúa sin conocimiento suficiente de los pasos que tiene que dar.

Planificación. En esta fase, los diálogos y las deliberaciones del sujeto consigo mismo están dirigidas a la definición de los objetivos que se desea alcanzar, a la selección de las estrategias, a la prevención de dificultades, al fomento de sentimientos de auto eficiencia en relación con las posibilidades de conseguir la meta propuesta, a la comparación de la tarea con los aprendizajes previos y a la motivación de la conducta hacia la meta.

De este modo, la planificación puede orientar el nivel de conocimiento y comprensión que se tiene de una tarea o tema que se va a trabajar o preparar; por ejemplo, si se hace una actividad sobre los diagramas de flujo, uno debe saber si conoce lo suficiente de ese tema, si han realizado previamente actividades de ese tipo, si los participantes conocen o no del tema, etc. Cada una de esas situaciones puede dividirse en dos alternativas, Si o No, la alternativa Si permite continuar con el siguiente paso, pues se sabe dominado (efectividad en tarea), mientras que, si no se sabe o se tienen dudas, puede plantearse realizar una acción correctiva, ya sea repasando, rehaciendo o rediseñando.

Preguntas orientadoras. Para la organización previa: ¿Cómo planificó las actividades de aprendizaje? ¿Prioriza los objetivos? ¿Qué objetivos quiero alcanzar? ¿Elabora un organizador previo a las actividades?

Control Consiste en la realización de la actividad y en el control que se ejerce sobre cada uno de los aspectos implicados en su desarrollo y sobre los posibles factores que pueden incidir en la concentración y distribución de los recursos. Sanz (2010 p.116), expresa que:

"el sujeto es capaz de revisar la adecuación de la estrategia que se está siguiendo, genera retroalimentación interna sobre cómo proceder, vigila el estado de ánimo, el interés y la ansiedad que suscita la realización de la tarea, coordina tiempos y mantiene la motivación, la seguridad personal y el esfuerzo, aunque encuentre dificultades para ello."

Por cuanto, el control de la propia actividad metacognitiva, denominada también regulación cognitiva, se basa en una secuencia de procesos de pensamiento mediante el cual se ponga en juego destrezas de planificación, supervisión y evaluación "de aquellos recursos cognitivos que se utiliza para aprender.

Preguntas orientadoras. A la atención selectiva. ¿Cómo ubico la información que requiero? ¿Cuáles son los detalles que me permiten retener los objetivos de la tarea? ¿Qué elementos me distraen? ¿Qué me motiva para atender la tarea y culminar mi objetivo? ¿Corresponde lo que planifico con lo que hago? ¿El diseño inicial de la planificación me permite reorientar las acciones? ¿Qué indicadores me permiten evaluarme durante el proceso?

Evaluación En este momento es cuando se verifica el proceso de aprendizaje, confirmando que se han cumplido los tiempos establecidos y valorando el modo de solución de las dificultades surgidas. Para Sanz (2010 p.158), es: "la evaluación implica comprobar los resultados de las propias acciones con los criterios previamente establecidos".

Preguntas orientadoras. Dirigidas a la reflexión. ¿Cómo reconozco efectivamente los procesos de aprendizaje en mí? ¿Cuál fue el proceso que fue más efectivo en otras situaciones de aprendizaje? ¿Dónde se presentó el mayor 
inconveniente para resolver una tarea? y ¿Cómo

puedo solventarlo en las actividades futuras?

Proceso de la estrategias metacognitivas de aprendizaje

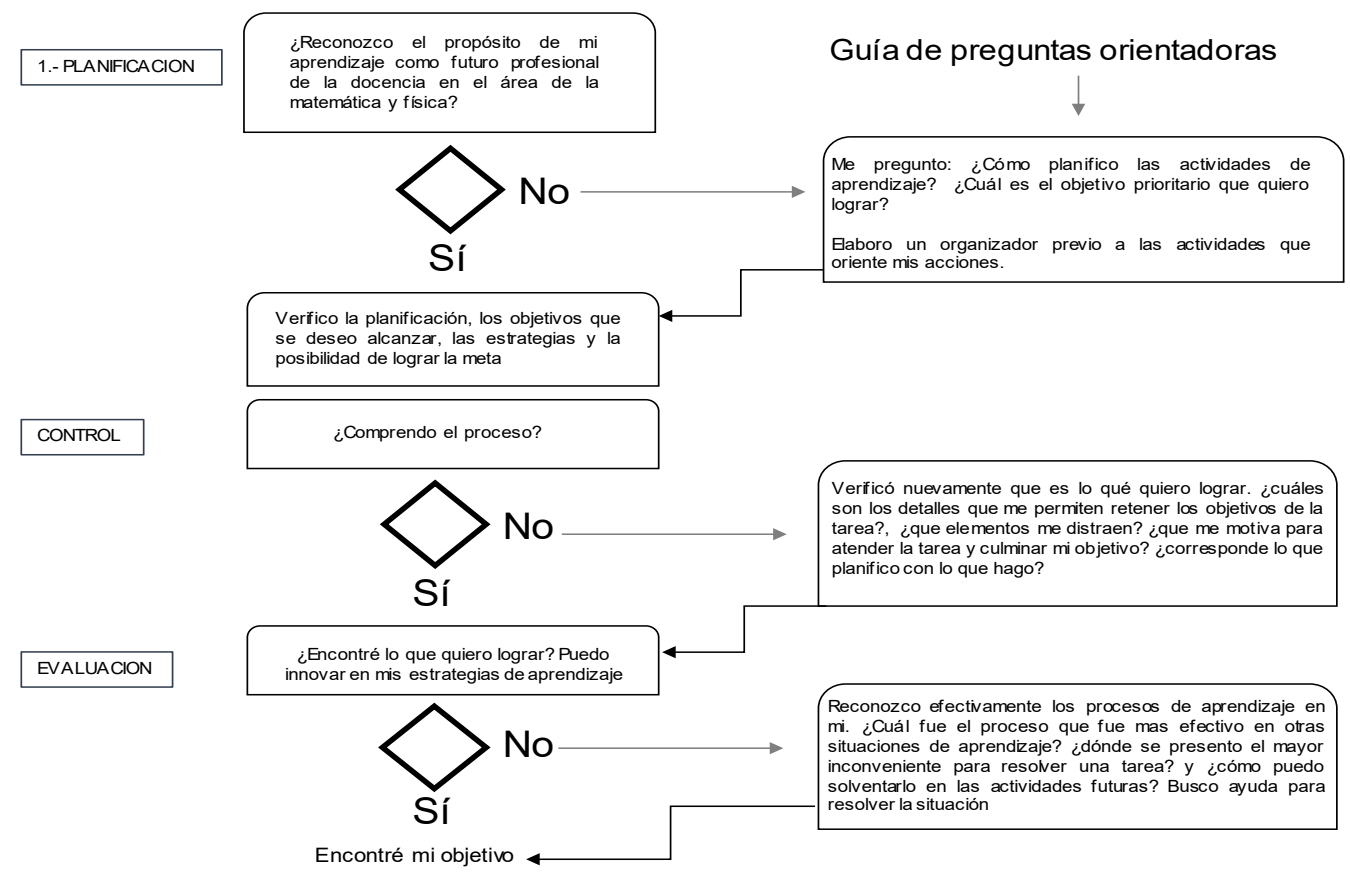

Figura 1 Proceso de las estrategias metacognitivas de aprendizaje

\section{CONCLUSIONES}

Para el objetivo número 1 referido a los tipos de competencias metacognitivas para el aprendizaje de los estudiantes universitarios de la licenciatura de matemática y física en la Universidad Popular del Cesar, se evidencia que el indicador con mayor promedio está representado por las competencias de planeación, lo cual refleja un alto nivel de consciencia por parte de los estudiantes sobre la importancia del uso de la planificación para su proceso de aprendizaje metacognitivo, sin embargo el indicador con menor promedio es competencias de autoevaluación de aprendizaje con 2.47 arrojando un resultado no favorable, lo que indica que los estudiantes requieren fortalecer sus procesos de autoevaluación para poder reflexionar sobre lo que están haciendo bien y lo que pueden mejorar, y así bajar los niveles de estrés condicionados por sus propias expectativas.

En relación al objetivo número 2 evidencia el componente metacognitivo con valor más bajo para el aprendizaje de los estudiantes universitarios de la licenciatura de matemática y física en la Universidad Popular del Cesar, referido al conocimiento declarativo con promedio de 2,60 y un resultado no favorable, quedando demostrado que las creencias que los estudiantes tienen sobre si mismos limitan su desarrollo académico profesional, originando desmotivación para adquirir nuevas habilidades; sin embargo, el conocimiento procedimental con un resultado de 3,67 de alto dominio indica que los estudiantes reconocen la ingente importancia 
de ceñirse a los procedimientos y normas a la hora de llevar a cabo las tareas asignadas.

Con respecto al objetivo número 3 relacionado con la propuesta de las estrategias metacognitivas para el aprendizaje de los estudiantes universitarios de la licenciatura de matemática y física en la Universidad Popular del Cesar, considerando los resultados evidenciados por las encuestas se sugiere la implementación de tres etapas, comenzando por planificar para orientar el conocimiento y la comprensión que tendrá el futuro profesional para ejecutar las tareas; seguido de un control que le permita redimensionar sus acciones cuando el producto final no sea el más funcional, terminando con la evaluación para que pueda validar el proceso de aprendizaje, así como el logro de los objetivos de forma efectiva, valorando el cómo soluciono los obstáculos presentados.

Ahora bien, con respuesta a la pregunta del objetivo general ¿De qué manera se desarrollan las competencias metacognitivas en los estudiantes universitarios de la licenciatura de matemática y física en la Universidad Popular del Cesa? Se pudo constatar que una vez que los estudiantes hacen consciencia que el desarrollo de sus competencias depende de su responsabilidad pueden apropiarse de herramientas que le permitan activar su proceso cognitivo para lograr los objetivos propuestos en su quehacer académico y profesional como futuros docentes que apalancan el crecimiento de una región y un país.

\section{REFERENCIAS BIBLIOGRÁFICAS}

Area, M.; Gros, B. y Marzal, M. A. (2008). Alfabetizaciones y TIC. . Síntesis.

Arias, F. (2006). El Proyecto de Investigación. Guía para su elaboración (Sexta ed.). Episteme.
Arias, W, Zegarra, J. y Velarde, O. (2014). Estilos de aprendizaje y metacognición en estudiantes de psicología de Arequipa. Liberabit, 20(2), 267-269. .

Arras A, Torres G y García-Valcácel. (2011). Competencias en tecnologías de información y comunicación de los estudiantes universitarios. . Revisa Latina de Comunicación, 66, pp. 1-23.

Botero et al. (2017). Pensamiento crítico, metacognición y aspectos motivacionales: una educación de calidad. Universidad Católica LuisAmigo.

Chávez, N. (2007). Introducción a la Investigación Educativa. La Columna. Tercera ed.

Correa, M. E.; Castro R. F. \& Lira R. H. (2003). Estudio descriptivo de las estrategias cognitivas y metacognitivas de los alumnos y alumnas de primer año de Pedagogía en Enseñanza Media de la Universidad del Bío-Bío. . Revista Iberoamericana de Educación. , Vol. 6 No. 3.

De Miguel, M. (2006). Métodos y Modalidades de enseñanza centradas en el desarrollo de competencias. . Alianza Universidad.

De Zubiría, J. (2007). Tratado de pedagogía conceptual Los modelos pedagógicos. Fondo de Publicaciones Bernardo Herrera Meriño.

Echeverría, R. (2014). Escritos sobre Aprendizaje. Santiago de Chile: Comunicaciones Nor-Este.

Flavell, J. (1996). El desarrollo cognitivo. Aprendizaje Visor. 
García, A. et al. (2006). La autoevaluación como actividad docente en entornos virtuales de aprendizaje/enseñanza. . RED. Revista de Educación a Distancia.

García, F. (2008). El cuestionario: recomendaciones metodológicas para el diseño de cuestionarios. ed. Limusa.

Goleman, D. (2013). Inteligencia emocional. . Editorial Kairos .

Hernández, Fernández y Baptista. (2010). Metodología de la Investigación. Mcgraw-HILL / Interamericana Editores, S.A. de C.V.

Hurtado. (2000). Metodología de la investigación holística. Instituto Universitario de Tecnologías Caripito.

Jimenes, M. (2020). Relación entre neurociencia y procesos de enseñanza-aprendizaje. Revista INFAD de Psicología.

Ledezma, F. (2010). Competencias metacognitivas, modelos expertos de resolución de problemas matemáticos y rendimiento académico de los estudiantes en la materia de cálculo I. Laboratorio de didáctica y Multimedia.

León, A., Risco, E. y Alarcón, C. (2014). Estrategias de aprendizaje en educación superior en un modelo curricular por competencias. Revista de la Educación Superior, (4) 172 pp. 123 - 144.

Martínez. (2018). Metodología delainvestigación. Editorial CEGAGE. Learning.

Mateos, M. (2001). Metacognición y Educación. Aique Grupo Editorial. .

Méndez. (2013). El desarrollo de niveles de competencias metacognitivas en la formación inicial de licenciados en pedagogía infantil. Amazonia investiga,
2 (1). Pp. 71-93.

Méndez, C. (2008). Metodología de Investigación Educativa. . Editorial Planeta.

Minesterio de Educación Nacional. (2003). Decreto 2566.

Minesterio de Educación Nacional. (2005). Decreto Nacional 2170.

Núñez, F. (2015). Estrategias de aprendizaje aplicables en estudiantes de Enfermería. . . Revista Electrónica de portalesmedicos.com.

Ormrond, J. E. (2008). Aprendizaje humano. Pearson educación SA.

PARRA, J. (2003). Guía de Muestreo.

Piña y Alfonzo. (2019). La metacognición en la educación universitaria. un caso de estudio. Revista Electrónica de Psicología Iztacala.

Sandoval, B. (2014). Perfil metacognitivo en estudiantes universitarios. Universidad Pedagógica Experimental Libertador . Revista de Investigacion y postgrado. ISSN: 1316-0087.

Sanz, M. (2010). Competencias cognitivas en Educación Superior. . Narcea, SA.

Shimamura, A. (2008). A neurocognitive approach to metacognitive monitoring and control. Psychology Press.

Tejada Fernández, J. y Navío Gámez, A. (2005). El desarrollo y la gestión de competencias profesionales: una mirada desde la formación. . Revista Iberoamericana 37 (02). , Pp. 1-16.

UNESCO. (2005). Hacia las sociedades del conocimiento. Informe Mundial de la UNESCO. Ediciones UNESCO. 